\title{
Self-learning and Self-repairing Technologies to Establish Autonomous Building Maintenance
}

\author{
Nuno D. Cortiços \\ Universidade de Lisboa/Faculdade de Arquitectura, Lisbon, Portugal
}

\begin{abstract}
Current maintenance models, applied to the building stock, struggle with the complexity and high costs associated with the necessary interventions to recover the original condition or repair the previous renovation works, related with the workers' experience, site conditions (logistic, climatic and environmental), technical skills, and experts' backgrounds. Thus, circumstances favor the introduction of monitoring by sensors, in active systems (ventilation, acclimatization, surveillance, fire-protection, et cetera), increasing efficiency and reducing operating costs. These goals are aligned with current requirements for passive construction solutions, considering the capacities of sensors and nano-technologies. All supported by Artificial Intelligence (AI), and its ability to learn and react, by monitoring the aging rate and external conditions impact on performance and conditioning the settings of renovation construction materials' and solutions' (future) composition. Nano-Technologies already proved their potential to transform the passive systems infinite structures, in traditional construction materials and solutions. By changing those into a semi-passive condition, able to react and adjust to adverse externalities slowing and/or inverting the performance losses. The research hypothesis an ecosystem to produce autonomous maintenance on buildings, through a digital condition assessment, on the actual system's components, with resort to nano-technology to reset those and trigger selfrepairing; to extend properties life-cycle and lifespan, and improve efficiency to maintain high performance, favoring the user's experience.
\end{abstract}

\section{Introduction}

In a planet with limited resources and a fast-growing population, it is crucial to ensure more efficient environmental interactions.

The Construction Industry Research and Development (R\&D) aims the overall performance, improving the energy efficiency and the technological integration of new functionalities driven by safety, lighter and faster construction and fewer maintenance needs, speeding up replacements (for newer solutions). On the other hand, Real State appeals to consumers by targeting innovation, using state-of-the-art technology, supported by mass production, to encourage consumption and product diversity. [1]

The Construction Sector plays an essential role both in society and in the economy, being responsible for 18 million direct jobs and 9\% of the GDP, in the EU alone. The Sector is a major consumer of goods and services from other areas of activity, especially raw materials, chemicals, and electric equipment, et cetera. Also, when structural changes are needed, the Sector tends to target the introduction of new policies regarding social conditions, climate changes or energy and financial efficiency. All supported by its economic capacity. [2]

Buildings are directly responsible for the most significant energy consumption share in the EU, around $40 \%$ of final energy, and emit about $35 \%$ of all greenhouse gases. The EU concerns comprise other types of impacts upon the constructed environment: indoor air quality, noise, and radioactive radiation. [2]

The Eurostat (EU-28) reports that the construction sector was responsible for $33 \%$ of the waste generated (2004-2012): 857,2 million tons per year, 1,6 tons per capita (2010). While, the households contributed to a $7 \%$ increase in waste production, over the same period [3]. Energy efficient buildings are so rare that EU demands a $90 \%$ cut in greenhouse emissions, by 2050 [4] [5] [6].

The Construction Sector is a conservative industry that avoids off-site assembly and technological innovations and tends to rely on hand labor skills. KPMG conducted a Global Survey on Construction Industry (2017) that showed that $72 \%$ of the engineering and construction executives believe that technological innovations will play a fundamental role in the Sector's future but less than half has a strategy to integrate those 
advances. Also reports that millennials, 20 to 24 years old (2015), represent (only) 7,3\%, of the workforce which means $10,7 \%$ less when compared with 2005 . In a Survey commissioned by Microsoft, 93\% of millennials assumed their preference for jobs where they are engaged with hitechnology.

With Modern Movement, the Sector gave the first steps to embrace technology by equipping buildings with nets of sensors and actuators to monitor conditions, mainly for fire safety, security control (surveillance and access), power supply, temperature, air quality (ventilation), et cetera. However, none was dedicated to surveying the construction materials and solutions condition.

The overall efficiency, in today's definition, depends on the knowledge and adaptability of technology to the circumstances. This research seeks to understand how to establish autonomous building maintenance with the contribution of self-learning machines and self-repairing technologies. Driven by maintenance's accuracy to promote energy efficiency and building performance in favor of the user's comfort and Institution's financial stability, while supporting the management teams and simplifying maintenance procedures, based on real-time information and passive reactions. Autonomous Maintenance System (AMS): sensors that monitor the parts condition, working in parallel with self-repairing construction materials and solutions to delay the natural degradation, while learning and sharing the data, with similar methods, to support and advise future actions and Sector players. In general, reducing energy needs, protecting the environment, ensuring public health and reinforcing public safety.

\subsection{Question}

On the airplane transportation century, transnational mobility and migration is a standard to major cities. The incomers tend to favor historical areas to live, twentycentury classic neighborhoods or consolidated districts, as an alternative. Therefore, properties prices rise, and new social problems emerge, which translate into pressure in the building stock update or use conversion and also few new constructions, following regulations, to reach the maximum sustainability and cost-benefit balance. [7]

In principle, modern building structures, made of reinforced concrete or profiled steel, can overcome the 120 years lifespan; therefore, constant maintenance must be considered for every case. In Western Europe, $80 \%$ of buildings will surpass 2050, mainly due to great historical legacy, architectonic value, low investment associated, and the high complexity of the operations necessary to demolish and initiate a new building process, plus bureaucracy. Although today's policies favor the renovation of the existing building stock; until now, only $1-2 \%$ received an update in conformity with the requirements imposed to new buildings, known as nearly zero-energy buildings (nZEBs). 2020 is foreseen as the year when even more restrictive measures will be imposed, for both new construction and existing stock. [7]

Contemporary construction shows an increasing tendency for automated assembly lines off-site, under a controlled environment (in south-east booming economies). Also, expectations are going further, pushing construction sites to a factory floor environment, robotized, autonomous and less and less dependent on humans. Despite this scenario, most of the finest, particular and support tasks will continue to rely on the workforce, as well as most of the maintenance-related tasks [8]. Even more, when it comes to inspection and maintenance operations, like conservation, rehabilitation or renovation. The goal is to endow those tasks with current technology to acquire and react, in real-time, to early-stage degradation problems, impossible to detect or approach under controlled costs: based on today's inspection practices and current tools.

Researchers have designed a similar approach, based on Building Information Modeling (BIM) by exchanging information with fire prevention and fire-extinguish devices, entitled "BIM-based Intelligent Fire Prevention and Disaster Relief System." The data collected is divided into five modules: Environmental Perception, Fire Sense, Locating/Evacuation, Locating/Fire Rescue, and Global Information. The system improves safety procedures management and minimizes risks to occupants in case of critical events [9]. However, the purpose of this research is different, since it intends to accelerate the maintenance procedures on early stages, as a self-defense/prevention mechanism: in this case, limited to the building's passive elements.

\subsection{Literature review}

The maintenance problems are diverse and complex, and buildings tend to have several owners, which leads to poor building systems management and lack of predicaments recognition, in the earlier stages. Therefore, mechanisms to foresee the building's life expectancy, with all its systems, need further development. [10]

Self-learning and self-repairing are two of the most promising technology's branches, capable of revolutionizing all industrial products unlike the traditional counterparts: justified by the capacity to adjust to diversities and fast R\&D investments' reimbursement, enabling competitive prices. [1]

The focus of industrial automation is on sensors technology, to achieve high levels of efficiency, through monitoring, controlling, and management of a set of operations. The Global Industrial Wireless Sensor Network (IWSN) has a projected growth of (up to) $15 \%$ per year, until 2020 [11]. Self-learning systems are gaining industry recognition and market share, as technology becomes more 'user-friendly' and prices decline. However, to be successful in the Sector, the outcome must match the manual labor in quality [12]. Especially relevant for maintenance works.

Nowadays, self-repairing induced by nanotechnology seems to offer the solutions to overcome systemic construction anomalies and enhance sustainability 
(reducing carbon emission and energy consumption) of 'traditional' materials and systems, by ensuring performance leverage [13]. Nano-materials can use contrarieties in their favor, such as harvesting radiation energy to start a new life-cycle [14].

The advances in technology lead to miniaturized electronic structures, reducing sensors to micro-scale, entering on "transducers" category, devices capable of transforming mechanical impulses into energy. The concept, known as MEMS, combines Micro, Electro, and Mechanical Systems under a silicon substrate, made by micro-fabrication techniques. The MEMS add to the unprecedented technological potential the low prices, a wide range of functionalities, abilities, portability, and ruggedness. [15]

Communications based on Wireless Sensor and Actuator Networks (WSANs) are growing its relation with Building Automation Systems (BASs) - limited to the active systems [16]. From the data confined to local hardware to cloud services shared online and accessible elsewhere. On this scale, only possible if WSAN embraces the latest low energy short-range wireless connectivity, empowered by the IoT, as Bluetooth Low Energy (BLE) or Bluetooth Smart [17].

Nevertheless, Building Automation Systems (BASs) and Wireless Building Automation (WiBA) are new technologies taking the first steps, obstacles or difficulties are known and under R\&D: expensive to install, update and commission. Since they depend on sensitive materials, running on low-power communications systems and highly exposed to external adversities. Issues related to the loss of connectivity, electromagnetic interference, and misreading are highly expectable. [18]

\subsection{Possible approach}

BIM, self-learning and self-repairing technologies play a fundamental role in the research: original data recording, tracking the aging process and triggering the self-repair process with Smart Materials and Solutions, set by previous learning.

On-Site Diagnosis (OSD), based on sensors, provides passive construction materials and solutions data to the BASs and WiBA, following and designing the degradation curve to pinpoint and alert to incoming failure while promoting maintenance actions on the tipping point, given by a cost-benefit schedule and driven by nanotechnology combined solutions. Assuring the first line of defense from the external elements, following the construction materials and solutions aging process and starting to repair and improve their lifespan and of the whole, ensured by a redundancy operation system, as presented below:

- waterproof sensor and self-repairing paint and coat systems in the envelope;

- humidity and temperature sensors and actuators to assure a low moisture presence between the envelope layers;

- humidity sensor and waterproof self-repairing systems in underground walls; and,
- others, as radiation counters and wind speed, to verify mechanical degradation in construction, need an aging marker tuning.

All data collected must be accessible to third parties, with codification security - in a world under threat, information about building condition can be sensitive shared in the cloud: enabling an ecosystem-based Internet of Things (IoT) objects. So, the data flux will contribute to Autonomous Maintenance Systems accuracy, towards Artificial Intelligence applied to Building Maintenance. The accessible data can be profitable in the future by the contribution of others in the development of new applications (software), by feeding statistical information and preventing accidents or validating behaviors.

The idea is not to replace the human-technical abilities to overview the construction materials and solutions condition but to provide redundancy based on autonomous surveillance performance access.

\subsection{Manuscript structure}

This manuscript considers other authors research work and advances to improve maintenance guidelines and strategies by proposing Autonomous Maintenance Systems. The manuscript is composed by an Introduction where the Building Stock is characterized, focusing on the demands imposed by current codes and regulations, technology potential to engage with the Sector and need to control maintenance costs. In the Question subchapter, the research purpose is framed from support to expected outcomes and the technology is succinctly described. In the Literature sub-chapter, the latest contributions on Autonomous Maintenance Systems (AMS) are approached, although none are overseeing the passive envelope systems. Possible Approach introduces the intent and how the know-how and technology can contribute to the described goal. The Methodology follows systematically the hypothesis advanced by the research, from the Sector technological stage through the aging and degradation monitoring and the system procedures. Results and Discussion approach the hypothesis of applying the fundaments of system technologies through three sensors developed to guarantee a precise work and capable of positive contributions to the accuracy of the AMS. The Conclusions present the advances expected for the Sector from the AMS first steps to the full operation and future research and developments.

\section{Methodology}

The Sector players are aware of the building stock degradation based on data acquired, which play a fundamental role to apply proper solutions in renovation, as shown by R\&D outcomes. So, why isn't constant care given to buildings? Critical reasons: the idea of buildings' robustness and the lack of awareness for parts and individual systems degradation to maintain the whole. Even when the condition is acknowledged, Institutions tend to favor other investments and delay the scheduled maintenance and only attend non-scheduled 
interventions, if and when the running operations are at risk, especially during crises periods.

One explanation relies on the diversity of construction degradation, mainly as structural and foundations settlement, high humidity levels, systemic or thermal cracks, pollution, biological and salts contamination, et cetera. Those can be read by the sensor's capabilities to collect, inform and follow preventive maintenance actions (i.e., renovation) or the actions induced by selfrepairing technology. Triggering measures as previously set, under a third-party energy source and, external conditions can provide diverse energy sources, e.g., radiation (UV, IR, and Gamma Rays), mechanical (seismic, rain and wind) and chemical (moisture and pollution), allowing the stabilization and stress release of construction materials and solutions. The same approach is already available under natural conditions, e.g., when the rain falls the paint layer is washed and pollutants removed. The process proposed is similar, once it takes advantage of the elements, on double duty, powered by nanotechnologies existent capacities, but instead of a 'generic solution for all' conditions, the system is set it to slow a specific aging process learned by the information gathered and compiled by the sensors at the spot.

By assessing sensors readings, it is possible to design a chart with construction materials and solutions' degradation and rate its performance. In real-time, triggering the pre-established measures to delay or stop functionality losses, caused by aging or external anomalies, preventing substantial 'contamination', nonanticipated costs and dissemination to other parts. The continuous evaluation of all measures promotes the selflearning to be used in future actions, leading to an organism capable of self-repairing its parts by favoring the whole and promoting the aimed user comfort and energy efficiency, as today demanded.

The whole ecosystem will manage 'big data,' only possible through the use of autonomous computational support and chemical industrial achievements, aligned with other areas of advance knowledge to Military and Civil uses. The Autonomous Maintenance (hypothesis) will become the ultimate reality only dependent on real test conditions. If their potential is proven, costs may be the only constraint for the implementation and execution of the entire ecosystem.

The sensors are the ecosystem providers, based in today's conventional technology, already available on many consumer's products, e.g., transport, wearables, communications, entertainment, security, health industry. Moreover, contributing to autonomous driving, orientation, safety, monitoring, maintenance (active systems), the interaction between human and natural conditions and proving their advantages with efficiency and accuracy. Some of the technologies mentioned, as self-learning and self-repairing, present a high cost on markets due to their novelty and the R\&D costs. Nevertheless, hi-tech solutions tend to have a rapid depreciation, just after one or two years, with massification, known as "The Golden Rule of Depreciation". [19]

Another key element is possibilities allowed by Bluetooth Low Energy (BLE) when come to connecting devices under a low radio frequency to spread a data beacon. With a minimum energy flow to transmit a beacon per day or less, in case of very adverse conditions, is possible to add auxiliary small-scale solar cells (under natural or artificial light), as small as $1 \mathrm{~cm}^{2}$. Nevertheless, the potential energy sources are diverse and can be harvest on-the-spot, as the electromagnetic already mentioned, plus dynamic, thermal, systemic movements, et cetera [20][21][22], collected by a slave device, as an energy supplier.

Solar cells can harvest an energy range between 2,0 to 5,5 Volts and, at the same time, use a battery backup to output from 1,1 to 5,2 Volts, allowing the sensor to spread BLE transmission to receivers on key-points (bridge and debug boards) based on an Ethernet or Wi-fi. Sensors and BLE or Wi-Fi net (IEEE 802.1) and selflearning technology should conceive models of aging/degradation to set future construction materials and solutions' to renovate the building components; based on quantity and quality data, the nanotechnology advances will drive to new engineering materials and products, in tune with local specifications and building needs.

In their development Autonomous Maintenance Systems must use the existent communications, Wi-Fi networks, internet connection, and cloud services, and promote the implantation of sensors in construction materials and solutions critical points, on the building: in the near future, a $\varnothing 12 \mathrm{~mm}$ by $55 \mathrm{~mm}$ deep hole will be the only intervention required for the insertion of an elongated cylindric capsule, with a sensor in the internal tip, tiny small solar panels, and communication antenna.

The self-learning and self-repairing systems do not work physically connected, but they are complimentary at each renovation cycle. First, collecting and processing previous information to provide alerts and analysis of degradation rates. Second, using nanotechnology knowledge to set and adjust new materials and solutions on building renovation, which allows the delay or reverses the aging cycles. The two combined, form the basis for autonomous maintenance, to be applied to a specific building or used to advise others under the same construction techniques.

The described Autonomous Maintenance can be improved if working side by side with other buildings, with similar construction materials and solutions on the proximity; when different, requires calibration. This wider network of buildings under an individual Autonomous Maintenance System should report the raw data and measure information to a secure cloud service to be shared with third parties for further R\&D. 'Helping' others AMS to interpret information to extend future construction materials and solutions' lifecycles and building's lifespan to favor users and property managers.

Establishing an AMS workgroup will permit to determine the redundancy validation, and guarantee the precise and justifiable incoming adjustments, leading to lower maintenance needs, while increasing performance, energy efficiency, and savings, that can be applied to a vast building stock. The use of survey technology in building active systems has already shown good results, ensuring better operation (increasing comfort and 
reducing running costs) and diminishing equipment maintenance procedures.

\section{Results and discussion}

The use of acquisition technology in building active systems has already presented good results, ensuring better operation (increasing comfort and reducing running cost) and diminishing equipment maintenance procedures. The same principles are replicable on envelope passive systems' components: responsible for $50 \%$ of energy consumption [23][24]. Everything gets worse when the condition is corrupted [25]. Nevertheless, self-learning technologies and self-repairing science need to be implemented separately, on construction and maintenance, and when proven reliable joint the Autonomous Maintenance ecosystem: to help the next generation properties managers, engineers and construction executives [26][27]. For that, educational Institutions need to be ahead and aware of the shifts in the Sector.

The construction materials and solutions are exposed to several 'attacks': chemical agents; mechanical strengths; galvanic corrosion; magnetic fields; and, thermal variations. Therefore, show a reduced life expectancy when compared with the conventional buildings' lifespan.

Construction Sensors and Smart Construction systems have already been applied to with success. The firsts measure a wide variety of data, such as thermal patterns, energy consumption, people and goods traffic, to improve systems performance related to safety and durability, as reinforced concrete superstructures. The seconds are engineered to react to environmental stimuli, like extraordinary temperatures, humidity, pressure levels, oxygenation presence, to promote the release of components or bacteria to start the healing process. [28]

Another key element is the possibility of micro and nanotechnologies measuring electric (multi-points) current by using the same power harvested for the transmission impulse of a passive radio frequency (BLE/Wi-Fi). Nanotechnologies can also contribute to Autonomous Maintenance ecosystem as sensors, as described, working on both phases. Collecting data on actual systems performance losses, to share with the nanotechnology suppliers, allowing them to set future materials and solutions in favor of a building's renovation.

\subsection{Application of smart CM\&Ss (Examples)}

\section{1) Self-repairing Paint Film/Coat}

In current construction, paint, varnish, and membrane coatings are the first barriers of defense from external aggressions. The main advantages are related with the simplicity of the tasks needed to apply the different coats (e.g., autonomous machinery is already set to do the job on constructions sites) and their competitive cost when compared to similar solutions. Nevertheless, their efficiency depends on the composition, application conditions and existing external aggressions. The predicted stability ranges between 5 and 6 years (or more) but often is shortened to 2 or 3 years, e.g., different resins percentages (20 to $40 \%$ ) or aqueous-dispersion type (acrylic or polyvinyl acetate) [29].

Their integrity is crucial for the construction strength and the overall performance, following the common anomalies: cracking, peeling, wrinkling, chalking, loss of coating shine [29].

One major issue with paint film results from the adhesive strength between the substrate and the coating [30]. Although, as shown by several researches, there is a natural correlation between quality and appearance [30] due to micro-cracks that jeopardize the coat performance. Paint film degradation can be related to solar exposure orientation, time-cycles, and incidence angular spectrum. By including two technologies setting a specific solution:

a) Self-learning - through a low resistant electric band painted over the coat (by replacing the limestone powder on the base of paint by metal particles); after the electric reads' calibration is possible to measure the mixture integrity and follow the aging deviation. So, if applied to the building's different envelope surfaces allows to perceive the distinct condition rates, assuming different maintenance life cycles and smart materials sets; temperature sensor or a radiation counter tuned to detect the substrate condition, as blisters under the coated base damage its adhesive capacity condition; and,

b) Self-repairing — by mixing binder nano-capsules with "fresh" resins or polymers composites on the tint solution, based on monitored data - if the capsule is produced with a binder similar to the original used in the paint, it will suffer the same aging process, which will cause it to break and release of a fresh binder, curing the anomalies resulting from decay and prolong the lifespan.

\section{2) Adaptive Plaster Subtract}

Many buildings are renovated with External Thermal Insulation Composite Systems (ETICS): thermal isolation above the original façade and covered by a dispersion of synthetic resins to prime the substrates for thin-layer plasters, putties, and paint coats. This mixture reinforced with fibers (glass, nylon, and polypropylene) are appealing for construction and renovation, mainly because their cost when durability, massive compressive strength, and stiffness are considered, on the other hand, are highly exposed to erosion, brittleness, poor tensile strength, and moisture movements compromising reality.

a) Monitoring — based on a sensor to read moisture and temperature, powered by (quarter dollar $/ 5$ euro cents coin size) solar panels - through a hole on the plaster layer it is possible to detect the presence of compromising conditions and, in time and on a daily basis, follow the degradation curve, alerting property managers to the weak state and providing the sets for the specific renovation composition; and,

b) Self-repairing — by a stimuli-responsive shape memory polymer, composites nanotubes, encapsulated on paint resins or polymers [31] 
the nanotubes when under a set temperature can dilate to allow the ventilation of the substrate materials, reducing the presence of moisture and stabilizing its rate, assuring the plaster strength and slowing the aging process.

3) Reactive Sodium Bentonite Barrier

The building stock, when in contact with the soil, tends - based on waterproof layers used on the construction - to reveal the presence of moisture, which demands complex and expensive maintenance works to stop exponential degradation, plus the uncertainty on efficiency and longevity. Usually, moisture appears in spots less reinforced, resulting in third parties/components anomalies, demanding extensive works until the confidence on the system is regained. The market provides multiple solutions based on synthetic or aqueous dispersion layers, resins or sodium bentonite, to fulfill the porous hydraulic mortars, but its success depends on simulated tests or real conditions, to be confirmed. Nevertheless, is possible to apply on the internal surface a layer of sodium bentonite or a similar aqueous resins film to block the moisture or water presence.

a) Monitoring - by embedding a sensor in contact with the sodium bentonite barrier allowing to detect the presence of moisture and register temperatures, for redundancy's propose and to establish a relationship between both conditions; is possible to install sensors (above described) powered by artificial light panels; and,

b) Self-repairing — through a base solution with sodium bentonite micro-capsules to extend the durability with efficiency- if a pre-cooked capsule shell, made with a similar sodium bentonite, dissolves if under the continuous presence of water, triggered as second or multiple stages; following the degradation of all layers which will provide a second efficient life-cycle, minimizing coming anomalies.

Technologies that proved their potentialities by transforming professional limitations into simple and mechanized tasks: from passive surveying to autonomous monitoring, by sensors working, full-time, highly precise reading and energy independent. From entirely passive systems under natural's degradation to semi-passive, by changing the infinite structure of traditional construction materials and solutions, empowered by previous knowledge collect on-site, capable of reacting and adapting to the adverse externalities, from the natural environment to human-made aggressions. The described technologies - as others already addressed by the research - allow extracting the full service from the construction materials and solutions, without compromising the overall building strength despite increasing the gap between maintenance cycles: cutting unnecessary costs, without compromising the components' performance.

Additional measures and strategies can achieve the redundancy of monitoring, as, e.g., if the technology cost is assumed, the sensors can be multiplied and placed on strategic parts (as independent units). So, in case of failure, others can check erratic readings, on-site or in the surrounding buildings, under similar systems. The same is true for self-repairing deployment if it is not dependent on third parties' action, the strategy is to assure that its activity is powered by the same sources that provoke the anomalies: nevertheless, with the sensors, on-site is possible to monitor their activities. So, by working together, it is possible to establish a controlled Autonomous Maintenance System for the existing building stock, as an ecosystem that reads, reports, advises and surveys.

The technology already proved, in other sectors, that autonomous systems can ensure performance and efficiency, at the same time. In the construction sector, implementation tends to spread to all phases; but the research is only focused on the last usage. Engaging between active and passive Autonomous Maintenance System can, in time, contribute to even greater building efficiency and performance, promoting the lifespan's extension, at lower running costs.

Research focuses on the hypothesis of introducing counter-aging measures, programmed through data acquisition; interpreted over Artificial Intelligence, to set and fine-tune the next renovation technologies. All to increment the performance, efficiency, and durability of each system to favor the building's condition: competitive running costs and to enhance the user's experience.

\section{Conclusions}

The building stock, especially in developed regions, will exist beyond 2050. Its renovation is under pressure, to comply with current environmental and economic concerns, as GHG emissions, energy efficiency, and floating energy prices. Maintenance methods play a crucial role to fulfill those goals. Nevertheless, automation has already started its path on building maintenance although limited to active systems, as, e.g., Building Automation Systems (BASs) and Wireless Building Automation (WiBA). This research is focused on the performance of passive envelope systems.

The existing maintenance models, applied to the building stock, are oriented to follow the aging and degradation processes with accuracy, considering workers' experience, site conditions (logistic, climatic and environmental), technical skills, and experts' backgrounds. Circumstances that justify the introduction of sensors' monitoring of active powered systems (ventilation, acclimatization, surveillance, fire-protection, et cetera), mainly, increasing efficiency and reducing running costs. These conditions are aligned with the presented approach to passive building elements, based on sensors' possibilities and nano and micro-technologies, improved by AI reports on previous maintenance solutions, applied and monitored, to support the future production of construction materials and solutions and, at the same time, assist managers' decision.

The research next step intends to test and certify the potential of the Autonomous Maintenance System, to confirm and minimize incoming risks and costs to the building pack, increasing efficiency and performance, working as an ecosystem capable of protecting itself from 
external aggressions. By sharing and comparing with third parties Autonomous Maintenance Systems data, located on similar buildings and conditions.

The first step is to install the (above mentioned) sensors on the same construction materials and solutions, to calibrate and collect data to draw the degradation charts and register the higher impact reactions cycles.

The second step will approach construction materials and solutions producers and partners, to incorporate second stage/self-triggering programmed measures to address anti-degradation, essential to justify sensors' monitoring.

The third step is to seek partners to design the architecture of a self-learning machine, to develop autonomous maintenance, capable of extended cycles empowered by future technology to achieve multi-stages rehabilitation reactions.

\section{Acknowledgment}

The author would like to thank: Fundação para a Ciência e Tecnologia (FCT - Portugal) for the financial support to the research project UID/EAT/04008/2013, through Centro de Investigação em Arquitectura, Urbanismo e Design, Faculdade de Lisboa, Universidade de Lisboa (CIAUD - Portugal).

\section{References}

1. Mounier, E., Fraux, R., Robin, L., \& Bonnabel, A. (2014, March 18). 6 and 9-axis DOF sensors are creating a new paradigm in the combos business Retrieved April 9, 2018, from eecatalog.com

2. European Commission. (2016). The European construction sector (Rep. No. Ref. Ares (2016)1253962). Brussels, March 11, 2016, from ec.europa.eu

3. Waste generation in EU-28 by type (2012). (2016, January 06). Retrieved April 09, 2018, from epthiktank.eu

4. European Parliament, Council, the European Economic and Social Committee and the Committee of the Regions, 'A Roadmap for moving to a competitive low carbon economy in 2050' (COM 112). Brussels 2011, from eur-lex.europa.eu

5. European Parliament and of the Council, Directive 2010/31/EU of 19 May 2010, on the energy performance of buildings, Energy Performance of Buildings Directive

6. European Parliament and of the Council, Directive 2012/27/EU, of 25 October 2012, on energy efficiency, amending Directives 2009/125/EC and 2010/30/EU and repealing Directives 2004/8/EC and 2006/32/EC, https://eur-lex.europa.eu

7. Smith, R. (n.d.). Berlin has the world's fastest rising city property prices. Retrieved April 17, 2018, from weforum.org

8. P. Vähä, T. Heikkilä, P. Kilpeläinen, M. Järviluoma, and E. Gambao, "Extending automation of building construction - Survey on potential sensor technologies and robotic applications," Autom. Constr., vol. 36, pp. 168-178, 2013. https://doi.org/10.1016/j.autcon.2013.08.002

9. M.-Y. Cheng, K.-C. Chiu, Y.-M. Hsieh, I.-T. Yang, J.-S. Chou, and Y.-W. Wu, "BIM integrated smart monitoring technique for building fire prevention and disaster relief," Autom. Constr., vol. 84, pp. 14 30 , 2017.

https://doi.org/10.1016/j.autcon.2017.08.027

10. V.I. Loganina, L.V. Makarova, R.V. Tarasov, "Method of assessment quality protective and decorative coating concrete cement," Case Stud. Constr. Mater., vol. 4, pp. 81-84, 2016.

11. TechNavio, Global Industrial Wireless Sensor Network (IWSN) Market 2016-2020, August, 2016.

12. Castro-Lacouture D. (2009) Construction Automation. In: Nof S. (eds) Springer Handbook of Automation. Springer, Berlin, Heidelberg. https://doi.org/10.1007/978-3-540-78831-7

13. J. E. Contreras and E. A. Rodríguez, "Nanostructured insulators - A review of nanotechnology concepts for outdoor ceramic insulators," Ceram. Int., vol. 43, no. 12, pp. 8545 8550 , 2017. https://doi.org/10.1016/j.autcon.2013.08.002

14. D. Papadaki, G. Kiriakidis, and T. Tsoutsos, "Chapter 11 - Applications of nanotechnology in construction industry," in Micro and Nano Technologies, A. Barhoum and A. S. B. T.-F. of N. Hamdy Makhlouf, Eds. Elsevier, 2018, pp. 343-370. https://doi.org/10.4018/978-1-5225-0344-6.ch004

15. Rosen, Y., \& Elman, N. (2012). Biomaterials science: An integrated clinical and engineering approach. Boca Raton: CRC Press / Taylor \& Francis Group.

16. L. Moldenhauer, R. Helmerich, E. Köppe, F. Haamkens, and J. Wittmann, "Experimental feasibility study about moisture in building materials measured with Bluetooth," Mater. Today Proc., vol. 4, no. 5, Part 1, pp. 5889-5892, 2017. https://doi.org/10.1016/j.matpr.2017.06.064

17. S. Raza, P. Misra, Z. He, and T. Voigt, "Building the Internet of Things with bluetooth smart," $\mathrm{Ad} \mathrm{Hoc}$ Networks, vol. 57, pp. 19-31, 2017. https://doi.org/10.1016/j.adhoc.2016.08.012

18. Z. Pang, H. Zhu, B. Xie, and M. Luvisotto, "Realtime and non-intrusive on-site diagnosis for commissioning wireless sensor and actuator networks in building automation," J. Ind. Inf. Integr., 2018. https://doi.org/10.1016/i.jii.2017.12.003

19. T. Gylfason and G. Zoega, "A golden rule of depreciation," Econ. Lett., vol. 96, no. 3, pp. 357362 ,

2007.

https://doi.org/10.1016/j.econlet.2007.02.008

20. J. Iannacci, "Internet of things (IoT); internet of everything (IoE); tactile internet; $5 \mathrm{G}$ - A (not so evanescent) unifying vision empowered by EHMEMS (energy harvesting MEMS) and RF-MEMS (radio frequency MEMS)," Sensors Actuators A Phys., vol. 272, pp. 187-198, 2018. https://doi.org/10.1016/j.autcon.2013.08.002 
21. R. Rondón, M. Gidlund, and K. Landernäs, "Evaluating Bluetooth Low Energy Suitability for Time-Critical Industrial IoT Applications," Int. J. Wirel. Inf. Networks, vol. 24, no. 3, pp. 278-290, 2017. https://doi.org/10.1007/s10776-017-0357-0

22. Qu, C. (2016). Piezoelectric Vibration Energy HarvesterActuated By Seismic Excitation (Master's thesis, Politecnico de Milano, 2016) (pp. 1-109). Milano: Politecnico de Milano.

23. C. Deb and S. E. Lee, "Determining key variables influencing energy consumption in office buildings through cluster analysis of pre- and post-retrofit building data," Energy Build., vol. 159, pp. 228-245, 2018. https://doi.org/10.1016/j.apenergy.2017.11.081

24. Cortiços, N., "Automation for building performance and maintenance efficiency," unpublished work, presented in Creative Construction Conference 2018, Ljubljana, July 1-2, 2018

25. J. Iwaro and A. Mwasha, "The impact of sustainable building envelope design on building sustainability using Integrated Performance Model," Int. J. Sustain. Built Environ., vol. 2, no. 2, pp. 153-171, 2013. https://https://doi.org/10.1016/j.ijsbe.2014.03.002

26. Handel, C., European Ventilation Industry Association (EVIA), Ventilation with heat recovery is a necessity in "nearly zero" energy buildings, HVAC Journal, Brussels. Available at: http://www.rehva.eu/index.php?id=239 Accessed: 2014-05-28, access on Nov, 6, 2017.

27. TechNavio - Infiniti Research Ltd., "Global Building Automation Software Market 2017-2021" London, 2017.

28. Sharp, S.R., Clemena, G.G. State of the art survey of advanced materials and their potential application in highway infrastructure, Charlottesville, Virginia Transportation Research Council; 2004.

29. T. V Uchaeva and V. I. Loganina, "Analysis of the risk at the finishing of the building products and construction of paint compositions," Case Stud. Constr. Mater., vol. 8, pp. 213-216, 2018. https://doi.org/10.1016/j.cscm.2018.01.001

30. M. A. Garrido, P. V Paulo, and F. A. Branco, "Service life prediction of façade paint coatings in old buildings," Constr. Build. Mater., vol. 29, pp. 394-402,

2012. https://doi.org/10.1016/j.conbuildmat.2011.10.057

31. H. Meng and G. Li, "A review of stimuli-responsive shape memory polymer composites," Polymer (Guildf)., vol. 54, no. 9, pp. 2199-2221, 2013. https://doi.org/10.1016/j.polymer.2013.02.023 